\title{
Predicting Mixture Effects over Time with Toxicokinetic- Toxicodynamic Models (GUTS): Assumptions, Experimental Testing, and Predictive Power
}

Sylvain Bart,* Tjalling Jager, Alex Robinson, Elma Lahive, David J. Spurgeon, and Roman Ashauer

Cite This: Environ. Sci. Technol. 2021, 55, 2430-2439

Read Online

ACCESS | Lill Metrics \& More | 回 Article Recommendations ｜ sl Supporting Information

ABSTRACT: Current methods to assess the impact of chemical mixtures on organisms ignore the temporal dimension. The General Unified Threshold model for Survival (GUTS) provides a framework for deriving toxicokinetic-toxicodynamic (TKTD) models, which account for effects of toxicant exposure on survival in time. Starting from the classic assumptions of independent action and concentration addition, we derive equations for the GUTS reduced (GUTS-RED) model corresponding to these mixture toxicity concepts and go on to demonstrate their application. Using experimental binary mixture studies with Enchytraeus crypticus and previously published data for Daphnia magna and Apis mellifera, we assessed the predictive power of the extended GUTS-RED framework for mixture assessment. The

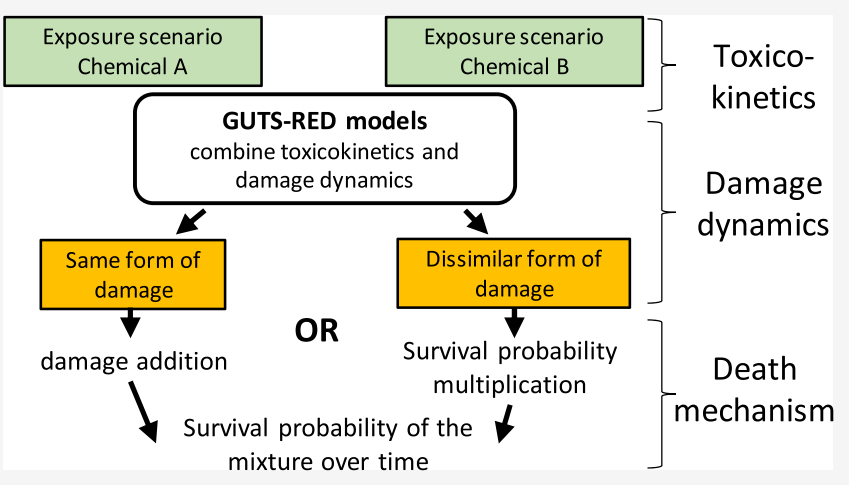
extended models accurately predicted the mixture effect. The GUTS parameters on single exposure data, mixture model calibration, and predictive power analyses on mixture exposure data offer novel diagnostic tools to inform on the chemical mode of action, specifically whether a similar or dissimilar form of damage is caused by mixture components. Finally, observed deviations from model predictions can identify interactions, e.g., synergism or antagonism, between chemicals in the mixture, which are not accounted for by the models. TKTD models, such as GUTS-RED, thus offer a framework to implement new mechanistic knowledge in mixture hazard assessments.

\section{INTRODUCTION}

Human activities release a plethora of chemicals into the environment ${ }^{1}$ that can lead to effects on nontarget organisms. The environmental risk assessment (ERA) for individual chemicals is established with robust methods, including experimental designs and data analysis methods in place. While risk assessment may take a chemical-by-chemical approach, in practice, ecosystems are subject to many inputs from agricultural, industrial, and domestic sources. These sources result in a wide range of mixture exposure scenarios that can affect nontarget organisms. ${ }^{3-5}$

The dominant approaches to predict mixture effects ignore the time dimension. ${ }^{5}$ As toxicity is a process in time, so is the action of mixtures. ${ }^{6}$ Therefore, mixture effect assessment needs diagnostic tools that account for these temporal aspects. To explain and predict the effects of mixtures, toxicokinetictoxicodynamic models (TKTD models), which simulate the time course of processes leading to toxicity, offer a promising approach. $^{7-9}$ Previous studies have presented TKTD models to analyze effects of mixtures on survival that have been successfully applied to mixture datasets. ${ }^{10-17}$

In the past decade, the development of the General Unified Threshold model for Survival (GUTS) framework firmly established the concept of damage dynamics, which takes place between the internal concentration and the effect. ${ }^{18,19}$ This concept is central to the GUTS framework as it provides an explanation for the time course of mortality, including cases where internal concentration kinetics fail. ${ }^{20,21}$ Recently, as the broad relevance of damage dynamics became clearer, this concept has been applied to DEBtox modeling for sublethal effects as well. ${ }^{22}$ To apply the toxicological assumptions of independently and jointly acting toxicants to TKTD models that simulate damage dynamics, a consistent mathematical framework is needed. Jager and Ashauer ${ }^{19}$ presented initial thoughts on how the GUTS framework can be used for the assessment of mixture effect. However, we do not know how to apply these ideas in practice, how well they work, and the predictive power of such models.

Received: August 6, 2020

Revised: December 3, 2020

Accepted: January 18, 2021

Published: January 26, 2021 
GUTS-RED independent action model

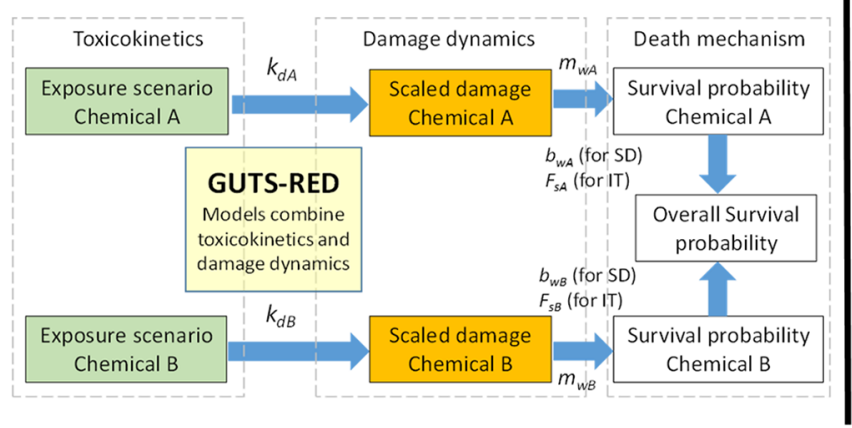

GUTS-RED damage addition model

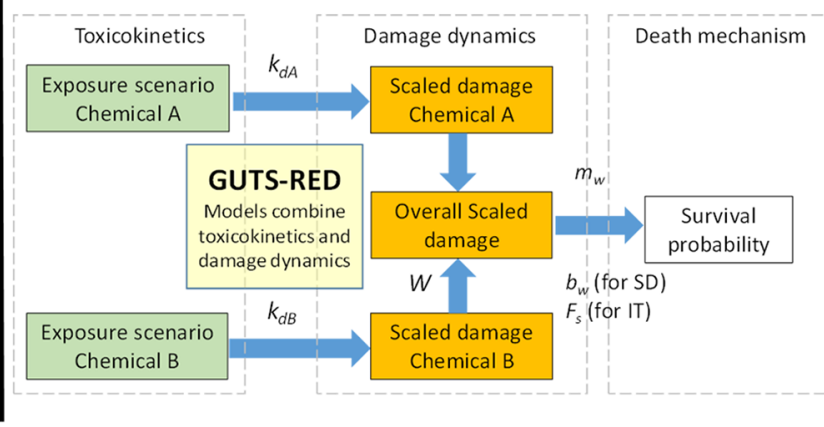

Figure 1. Description of the GUTS-RED mixture models under the assumption of independent action (left panel) and same mode of action (or same form of damage) (right panel). $k_{\mathrm{d}}$ is the dominant constant rate, $m_{\mathrm{w}}$ is the median of the threshold distribution, $b_{\mathrm{w}}$ is the killing rate (when considering the stochastic death approach, $\mathrm{SD}$ ), and $F_{\mathrm{s}}$ is the fraction spread (when considering the individual tolerance approach, IT). $W$ is a weight factor that normalizes the scaled damage of chemical B to chemical A. The models are illustrated for a binary mixture but can be expanded to mixtures with more components.

Here, we extend the GUTS framework, and specifically, the GUTS reduced (GUTS-RED) models, for application to mixture effects over time. GUTS-RED combines toxicokinetics and damage dynamics into a single compartment and therefore links external concentrations to the effect on survival. GUTSRED models are relatively simple with a few parameters. Hence, they are efficient models for effect assessment because they require a few data, no measurement of body residues, and no other toxicokinetic information. ${ }^{23}$ To extend GUTS, we first translate the classical mixture effect assumptions into equivalent mechanistic concepts to be implemented into the equations of the GUTS-RED models. We then test the extended model assumptions and assess their predictive power by using bespoke mixture effect data and mixture experimental results from the literature.

\section{MATERIALS AND METHODS}

Toxicokinetics-Toxicodynamics. A Brief Background of the GUTS Framework. The GUTS models simulate the time course of processes leading to the death of an organism. $^{18,19}$ They account for the accrual of, and recovery from, damage (toxicodynamics, TD), which forms due to the bioaccumulation, distribution, biotransformation, and elimination of the chemicals in the organisms (toxicokinetics, TK). In the absence of information on body residues (measurements or predictions), the TK and TD cannot be modeled separately, and the GUTS reduced (GUTS-RED) models, which combine the TK and TD, are used instead. In GUTS-RED models, the dominant rate constant $k_{\mathrm{d}}$ describes the dynamics of the "scaled" damage and will represent the one-compartment approximation of the "true" two-compartment behavior (TK and damage dynamics). To describe the death mechanism related to the damage, two causations of the process affecting survival are formalized: the stochastic death (SD) and individual tolerance (IT) approaches. The SD approach assumes that individuals are identical and have a probability to die upon chemical stress, which increases with increasing damage once some threshold damage has been exceeded. The IT approach assumes that individuals have differences in their sensitivity to chemical stress, and when the damage exceeds an individual's threshold, it dies instantly. Both approaches can lead to different data interpretation and predictions for the time course of effect. ${ }^{21}$ Consequently, we here use both approaches for mixture hazard assessment, and because the
GUTS framework provides consistent mathematical formulations for both, we build mixture models within that framework.

Extension of the GUTS Model for Mixture Toxicity. In GUTS, exposure to a chemical leads to damage that in turn leads to effects on survival. According to this assumption, mixtures of two chemicals can lead to two basic possibilities: the chemicals lead to the same or dissimilar forms of damage. The first option is appropriate for chemicals with the same mode of action and possibly also for chemicals acting on the same physiological process (e.g., insecticides acting on the same aspect of the nervous system). In this situation, the damage produced by each chemical can be added up, and this is referred to as the GUTS-RED (scaled) damage addition (DA) model in this paper (Figure 1). For chemicals leading to dissimilar forms of damage, the assumption is that the effects of both chemicals are independent and that we need to multiply their effects (i.e., the survival probabilities). This second possibility is referred to as the GUTS-RED independent action (IA) model (Figure 1).

When damage addition applies to a mixture, the damage dynamics of each chemical is still characterized by its own dominant rate constant $\left(k_{\mathrm{d}}\right)$. Hence, each substance in the mixture has different damage dynamics, but because the type of damage produced is the same, they can be summed. For damage addition to apply, the components in the mixture must share parameters linking damage dynamics to survival $\left(m_{\mathrm{w}}, b_{\mathrm{w}}\right.$, and $\left.F_{s}\right)$. Because chemicals can differ in their efficiency in causing damage, and because we worked with scaled damage, an additional model parameter, the weight factor $W$, needs to be applied to the substance-specific attributed scaled damage before these are summed. This is similar to the concept of relative potency for mixture effects, which has been successfully used in the past for in vitro bioassays, ${ }^{24}$ polychlorinated dibenzodioxins (toxic equivalency factors), ${ }^{25}$ water quality criteria for herbicides with the same mode of action in surface water, $^{26}$ and QSAR models considering concentration addition. $^{27}$ The $W$ factor is a new parameter that is constant over time, and it is fitted during the simultaneous fit of the single exposure data of both substances with the GUTS-RED damage addition model.

The IA model is based on the assumption that the chemicals act on different target sites, affecting different physiological processes and, thus create different damage forms. Consequently, the two chemicals have their own independent sets 


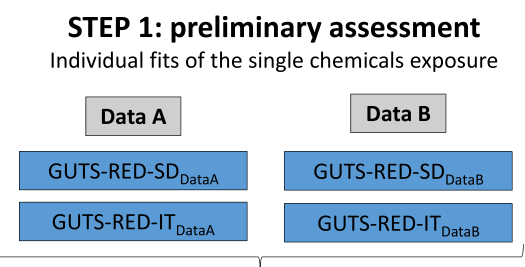

Plot the mortality parameters (Figure 3)

\section{STEP 2: preliminary assessment}

Simultaneous fits of the single chemicals exposure

Data A + Data B

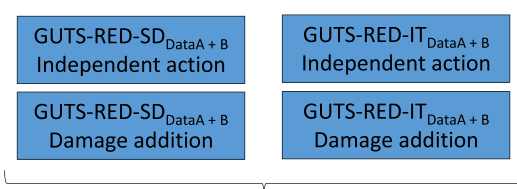

Comparing of the simultaneous fits between the independent action model and the damage addition model with the likelihood-ratio test (Table 1)
STEP 3: ultimate test

Prediction of the mixture effects and comparing with the mixture data

Mixture Data AB

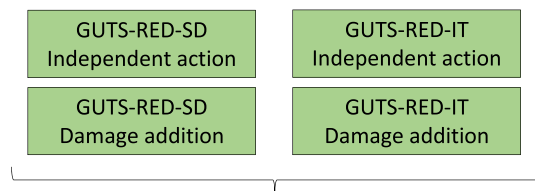

Compare AIC and R2 (Table 1, Figure 4B, 5B, 6, S1B, S2, S3B, S4, S5, S6, S7)

Figure 2. Work flow for data analysis with the GUTS independent action and damage addition models to identify the most appropriate model and assumptions.

of GUTS parameters, and the survival probabilities are multiplied to create an overall survival probability due to the mixture effect.

The equations of the GUTS-RED mixture models are derived from the GUTS framework fully presented in Jager et al. ${ }^{18}$ The full set of equations for both mixture models can be found in the Supporting Information.

Choice and Assessment of the Two GUTS-RED Mixture Model Fits and Predictions (DA and IA). For most chemicals, it will not be known if they will create the same or dissimilar forms of damage. Studying the adverse outcome pathway, at the subindividual level, on chemical effects can lead to insights. ${ }^{28}$ For example, for chemicals known to act on the same target, it is expected that the DA model provides better results. However, for many chemicals, we have insufficient knowledge about the type of damage they cause.

The application of the DA model implies that the related mortality model parameters, i.e., those linking the sum of scaled damage to survival, are shared by the chemicals in the mixture: $m_{\mathrm{w}}, b_{\mathrm{w}}$, and $F_{\mathrm{s}}$. This offers a possibility to check if the DA model is appropriate for mixture applications even before performing mixture tests. The parameter values $\left(m_{\mathrm{w}}, b_{\mathrm{w}}\right.$, and $F_{s}$ ), derived by calibrating the GUTS-RED model to data from each substance separately, can be examined to explore the possibility that chemicals share the same value of the model parameters. Considering IT, the threshold $m_{\mathrm{w}}$ provides no information on whether the DA model is possible, but the parameter $F_{s}$ does because if chemicals induce the same form of damage, the distribution of tolerances across individuals to these damages must be the same. Therefore, $F_{s}$ should be similar (or at least the CIs should overlap). Considering SD, the product $m_{\mathrm{w}} \times b_{\mathrm{w}}$ should be similar (or at least an overlap of their CIs) because in GUTS models, we do not know the true level of damage, and it is usual to work with the scaled damage, meaning that the damage levels are divided by the unknown partition coefficient between internal concentration and damage. ${ }^{19}$ Therefore, $m_{\mathrm{w}}$ and $b_{\mathrm{w}}$ have the unit of the external concentration and differ from the "true" $m_{\mathrm{w}}$ and $b_{\mathrm{w}}$ by a factor: the partition coefficient. This unknown value of the partition coefficient returns as a factor by which $m_{\mathrm{w}}$ is divided and $b_{\mathrm{w}}$ is multiplied, and hence, their product should be the same for additive chemicals. Therefore, the first step (Figure 2) is to plot the single substance-derived $F_{\mathrm{s}}$ for IT and $m_{\mathrm{w}} \times b_{\mathrm{w}}$ values for SD to evaluate if it is appropriate to use the DA model for the considered mixture. If the plotted CIs for the parameters for the individual substances overlap, then DA is a possibility to consider; if they do not overlap, then DA is not appropriate.
In a second step (Figure 2), for all tested binary mixtures, we fitted simultaneously the two single exposures with the DA and IA models. It is important to note that the log-likelihood of a simultaneous fit for two chemicals with the IA model will be equal to the sum of the log-likelihoods of fits for the single chemicals separately because no parameters are shared. The simultaneous fit of the single exposures with the DA model is more interesting since parameters need to be shared between the compounds. Because the DA model is, when only considering single exposures, nested in the IA model, this allows for a formal statistical test to assess if the DA model is a possibility (the sum of the log-likelihoods for the independent fits can be compared to the simultaneous fit in a likelihoodratio test).

The third step, and ultimate test (Figure 2), is in the prediction of the mixture effect with both the IA and DA models. Since the IA and DA models, applied to mixtures of chemicals, are not nested, their predictions cannot be compared in a formal likelihood-ratio test. Instead, they are compared more qualitatively by their $r$-square $\left(r^{2}\right)$ value and the Akaike information criterion (AIC). We hypothesize that these three steps together provide information on whether chemicals share the same mode of action and allow model selection for the prediction of mixture effects.

Test Organism, Experimental Design, and Chemicals. Enchytraeus crypticus (Enchytraeidae; Oligochaeta; Annelida) were originally sourced from the laboratory of the Department of Ecological Science, Vrije Universiteit, Amsterdam, The Netherlands, and were maintained in culture at the UK Centre for Ecology and Hydrology, Wallingford (UK). For all experiments, adult individuals were exposed at $15{ }^{\circ} \mathrm{C}$ in the darkness, in $1 \mathrm{~mL}$ of artificial fresh water ${ }^{29}$ in 24 -well plates, with one individual per well ( 24 animals per treatment), for 96 h. Survival was monitored at 3, 6, 24, 48, 72, and 96 h for a total of seven time points. Individuals were classified as dead if they did not respond to repeated touching with a pin.

To test the IA model, we selected two chemicals with different modes of action for which preliminary data indicate that there was no interaction. The first is MGK 264 (CAS: 113-48-4), and the second is glyphosate (CAS: 1071-83-6), both obtained from Sigma Aldrich (St. Louis, MO, US). To test the DA model, we selected two fungicides from the same class that have the same putative mode of action and without any potential interaction (based on preliminary experiment): prochloraz (CAS: 67747-09-5) and triflumizole (CAS: 6869411-1), both obtained from Santa Cruz Biotechnology (Dallas, TX, US). Both fungicides inhibit the sterol biosynthesis in 


\section{Expected independent action}

\section{Expected Damage addition}
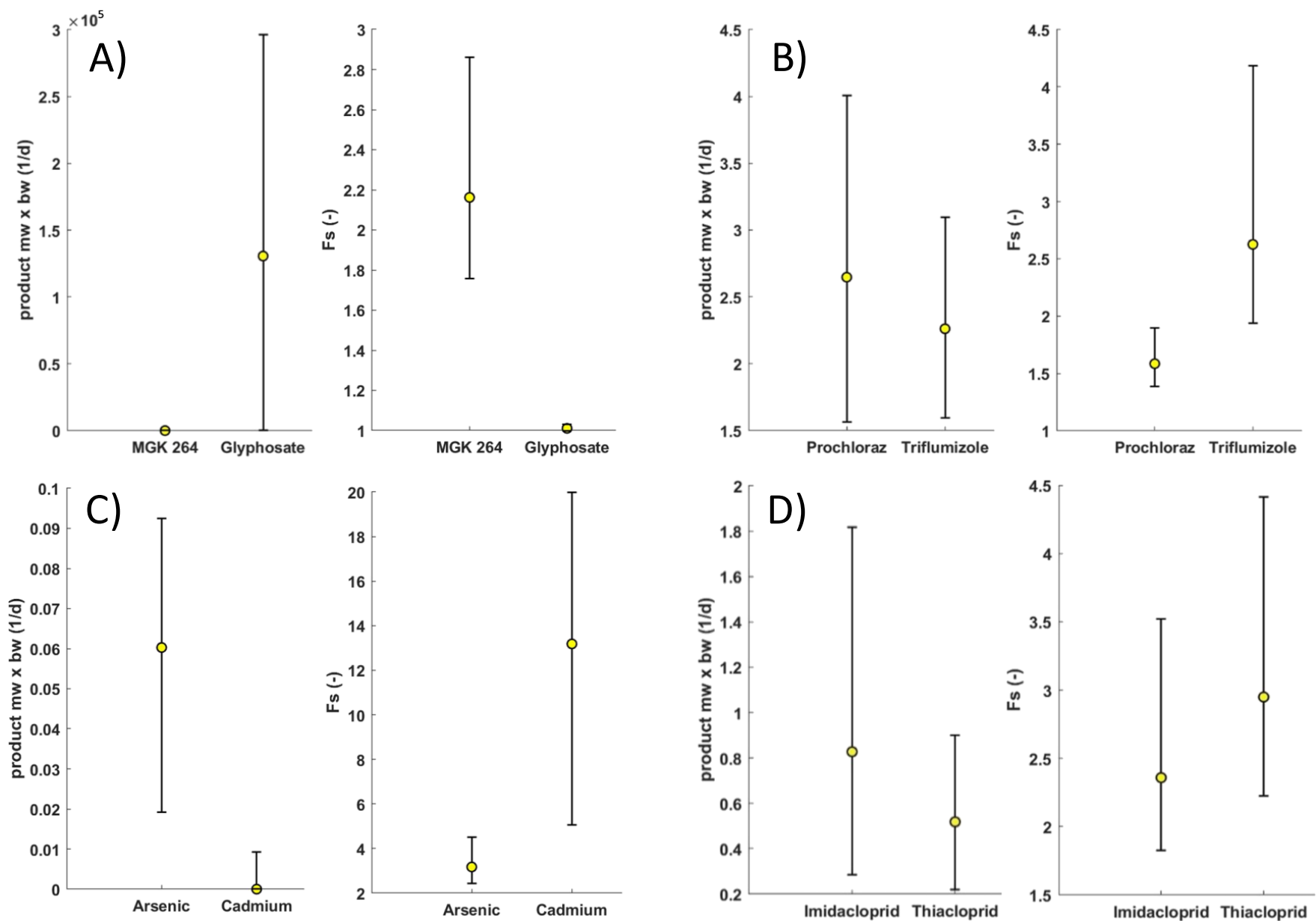

Figure 3. Comparison of the GUTS mortality-related parameters between (A) MGK 264 and glyphosate, (B) prochloraz and triflumizole, (C) arsenic and cadmium, and (D) imidacloprid and thiacloprid. For each comparison, the left plot is for the stochastic death approach (SD) with the product of the median of the threshold distribution $\left(m_{\mathrm{w}}\right)$ and the killing rate $\left(b_{\mathrm{w}}\right)$, and the right plot is for the individual tolerance approach (IT) with the fraction spread $\left(F_{s}\right)$. The yellow point is the best-fit value with the $95 \%$ confidence intervals. For (A), the value for MGK 264 is 0.59 (CI, $0.31-2.58)$, and for glyphosate, the value is $2.03 \times 10^{5}$ ( CI, 172 to $\left.2.96 \times 10^{5}\right)$. For the GUTS-RED damage addition model to be used, the two chemicals need to share their GUTS mortality parameters and thus need to show overlap of the parameter samples, as in (B) and (D). If no overlap is observed, then the GUTS-RED independent action model is more appropriate, as in (A) and (C).

membranes and act on the same target site $(\operatorname{erg} 11 / \text { cyp } 51)^{30}$ and the target site is present on the genome of E. crypticus. ${ }^{31}$

Based on preliminary testing and results, we selected a range of concentrations for testing expected to lead to effects on survival (Table S1). The single exposures (one chemical) were designed to calibrate the GUTS-RED mixture models and to predict the binary (two chemicals) mixture effects. Therefore, we chose concentrations to cover the toxic effect from no effect to $100 \%$ mortality. According to the toxicity profile and the expected recommended model used for the prediction of the mixture (DA or IA), we next selected a relevant range of concentrations in the mixture experiment. For the two fungicides (DA), we exposed individuals to half of the concentration of each fungicide as used in the single exposure (e.g., single exposure: $10 \mathrm{mg} \mathrm{L}^{-1}$, binary mixture: $5+5 \mathrm{mg} \mathrm{L}^{-1}$ of each fungicide; Table S1). For the assessment of the IA model, glyphosate showed a strong threshold effect, meaning that once the concentration exceeds the threshold, the effect on survival was high ( $100 \%$ mortality), while MGK 264 showed a slow increase in the effect with exposure concentration. Based on these preliminary results, we chose to increase the concentration of these two chemicals in the mixture but kept the glyphosate concentration under the threshold. If the hypothesis of independent action is correct, then the addition of glyphosate should not increase the expected effect due to MGK 264. Single and mixture exposures were performed at the same time to minimize interexperimental variability. Finally, to further test the two different GUTS-RED mixture models, we extracted suitable available data from the literature. We used data from Robinson et al. ${ }^{32}$ who exposed the western honeybee Apis mellifera to single and mixture treatments of arsenic and cadmium (see also Hesketh et al. ${ }^{33}$ for more details) and data from Loureiro et al. ${ }^{34}$ who exposed Daphnia magna to single and mixture treatments of imidacloprid and thiacloprid. All concentrations were translated into $\mu \mathrm{M}$ units in the model for more consistency especially for the DA model, which adds up the damage with an external concentration unit.

Model Calibration and Prediction. All calculations were performed in Matlab 2020a. The Wilson score interval was 
used to express the uncertainty in the survival data, which allows confidence intervals (CIs) to be plotted for each data point. ${ }^{35}$ The DA and IA models were implemented in the BYOM modeling platform (www.debtox.info/byom.html). The optimization of the parameter values was performed with the parameter space explorer. ${ }^{36}$ This algorithm is optimized for GUTS analyses and combines grid search, a genetic algorithm, and likelihood profiling, giving the CIs of the parameter values. To produce CIs on the model curve, a sample from the parameter space explorer is used. The parameter space explorer was also used to produce CIs for the product of $b_{\mathrm{w}} \times m_{\mathrm{w}}$ (Figure 3): the algorithm returns a sample from the parameter space for error propagation. Then, the product $b_{\mathrm{w}} \times m_{\mathrm{w}}$ is calculated for each element of this sample and used as a new sample, and the edges of this new sample are the CIs for the product. This is fully analogous to the CIs on model predictions as explained by Jager. ${ }^{36}$

For all calibrations, the background mortality $\left(h_{\mathrm{b}}\right)$ was fitted to the survival in the control treatment and kept fixed while fitting the toxicity parameters. For all fits and predictions, we provided the model efficiency with the $r^{2}$ (i.e., observed vs predicted) and Akaike information criterion (AIC) value. For the calculation of the AIC for the prediction, we used the number of toxicity parameters ( 5 for DA and 6 for IA).

\section{RESULTS AND DISCUSSION}

Using TKTD Evidence to Provide Information on the Chemical Mode of Action. The comparison of the mortality-related parameters for the chemicals is not only useful to choose between the DA and IA but also as a powerful tool to explore whether two chemicals may share the same mode of action. Essentially, this analysis helps one understand if it is possible that different toxicants cause the same form of damage. For all tested mixtures showing no overlap in their CIs of the mortality parameters (Figure 3), the simultaneous fit on the single exposures was significantly better with the IA model (Table 1). This supports the conclusion that the DA model is not applicable for the mixture. For the prochloraz and triflumizole experiment, the simultaneous fits of the single exposures with the DA model were good (Figure 4A and Figure S1A) and consistent with the putative similar forms of damage; even if for the IT approach, the simultaneous fits of the single exposures were significantly better with the IA model (Table 1). It is important to note that the simultaneous fit of the single exposures with the IA model will always result in the best fits (in terms of MLL) because the chemicals are independent of their own GUTS parameters. In other words, the fits of the IA model are equivalent to the fits on the single exposures taken separately. In contrast, the DA model forces the different chemicals to share parameters, which constrains the model behavior, and thus always leads to comparatively worse fits. For this fungicide mixture, the prediction from the DA models for combined exposure, with both the SD and IT approaches (Figure 4B, Figure S1B, and Table 1), surpassed those for the IA models, which considerably underestimate the observed effects (Figure S2 and Table 1). The plot of the mortality parameters, the simultaneous fit of the single exposures, and prediction for the mixture effects together identify the DA model as more appropriate for this mixture with putative similar mode of action, consistent with our underlying theory for model development. At this stage, a mixture toxicity test is required to deliver the ultimate proof about the chemical mode of action. However, the analyses of
Table 1. Assessment of the Simultaneous Fits of the Single Exposures and the Predictions of the GUTS-RED-SD and IT Independent Action (IA) Model and the GUTS-RED-SD and IT Damage Addition (DA) Model for the Binary Mixtures: Prochloraz (PCZ) and Triflumizole (TRI), MGK 264 (MGK) and Glyphosate (GLY), Arsenic (As) and Cadmium (Cd), and Imidacloprid (IMI) and Thiacloprid $(\mathrm{THI})^{a}$

\begin{tabular}{|c|c|c|c|c|c|}
\hline mixture & models & \multicolumn{4}{|c|}{ assessment } \\
\hline \multicolumn{2}{|c|}{ simultaneous fits on single exposures } & \multicolumn{2}{|c|}{$\begin{array}{l}\text { likelihood-ratio } \\
\text { test (IA vs DA) }\end{array}$} & \multicolumn{2}{|c|}{ conclusion } \\
\hline \multirow[t]{2}{*}{ PCZ + TRI } & $\begin{array}{l}\text { GUTS-RED-SD-IA; } \\
\text { GUTS-RED-SD-DA }\end{array}$ & \multicolumn{2}{|l|}{0.6101} & \multicolumn{2}{|c|}{$\begin{array}{l}\text { DA is } \\
\text { possible }\end{array}$} \\
\hline & $\begin{array}{l}\text { GUTS-RED-IT-IA; } \\
\text { GUTS-RED-IT-DA }\end{array}$ & \multicolumn{2}{|l|}{0.00433} & \multicolumn{2}{|c|}{$\begin{array}{l}\text { DA not } \\
\text { supported }\end{array}$} \\
\hline \multirow[t]{2}{*}{ MGK + GLY } & $\begin{array}{l}\text { GUTS-RED-SD-IA; } \\
\text { GUTS-RED-SD-DA }\end{array}$ & \multicolumn{2}{|c|}{$<2.2 \times 10^{-16}$} & \multicolumn{2}{|c|}{$\begin{array}{l}\text { DA not } \\
\text { supported }\end{array}$} \\
\hline & $\begin{array}{l}\text { GUTS-RED-IT-IA; } \\
\text { GUTS-RED-IT-DA }\end{array}$ & \multicolumn{2}{|c|}{$<2.2 \times 10^{-16}$} & \multicolumn{2}{|c|}{$\begin{array}{l}\text { DA not } \\
\text { supported }\end{array}$} \\
\hline \multirow[t]{2}{*}{ As $+\mathrm{Cd}$} & $\begin{array}{l}\text { GUTS-RED-SD-IA; } \\
\text { GUTS-RED-SD-DA }\end{array}$ & \multicolumn{2}{|l|}{0.004427} & \multicolumn{2}{|c|}{$\begin{array}{l}\text { DA not } \\
\text { supported }\end{array}$} \\
\hline & $\begin{array}{l}\text { GUTS-RED-IT-IA; } \\
\text { GUTS-RED-IT-DA }\end{array}$ & \multicolumn{2}{|l|}{0.004053} & \multicolumn{2}{|c|}{$\begin{array}{l}\text { DA not } \\
\text { supported }\end{array}$} \\
\hline \multirow[t]{2}{*}{$\mathrm{IMI}+\mathrm{THI}$} & $\begin{array}{l}\text { GUTS-RED-SD-IA; } \\
\text { GUTS-RED-SD-DA }\end{array}$ & \multicolumn{2}{|l|}{0.4386} & \multicolumn{2}{|c|}{$\begin{array}{l}\mathrm{DA} \text { is } \\
\text { possible }\end{array}$} \\
\hline & $\begin{array}{l}\text { GUTS-RED-IT-IA; } \\
\text { GUTS-RED-IT-DA }\end{array}$ & \multicolumn{2}{|l|}{0.3482} & \multicolumn{2}{|c|}{$\begin{array}{l}\mathrm{DA} \text { is } \\
\text { possible }\end{array}$} \\
\hline mixture & models & \multicolumn{4}{|c|}{ assessment } \\
\hline \multicolumn{2}{|c|}{ prediction of the mixture } & $\begin{array}{l}\text { AIC (IA } \\
\text { vs DA) }\end{array}$ & & & $\begin{array}{l}\text { best } \\
\text { nodel }\end{array}$ \\
\hline \multirow[t]{2}{*}{$\mathrm{PCZ}+\mathrm{TRI}$} & $\begin{array}{l}\text { GUTS-RED-SD-IA; } \\
\text { GUTS-RED-SD-DA }\end{array}$ & $\begin{array}{r}937.16 ; \\
292.4\end{array}$ & $\begin{array}{r}0.16 ; \\
0.9\end{array}$ & & $\mathrm{DA}$ \\
\hline & $\begin{array}{l}\text { GUTS-RED-IT-IA; } \\
\text { GUTS-RED-IT-DA }\end{array}$ & $\begin{array}{l}393.88 ; \\
\mathbf{2 6 9 . 8 7}\end{array}$ & $\begin{array}{r}0.54 \\
0.9\end{array}$ & & DA \\
\hline \multirow[t]{2}{*}{ MGK + GLY } & $\begin{array}{l}\text { GUTS-RED-SD-IA; } \\
\text { GUTS-RED-SD-DA }\end{array}$ & $\begin{array}{r}338.62 ; \\
2262.9\end{array}$ & $\begin{array}{r}0.91 \\
(-\end{array}$ & & IA \\
\hline & $\begin{array}{l}\text { GUTS-RED-IT-IA; } \\
\text { GUTS-RED-IT-DA }\end{array}$ & $\begin{array}{l}331.51 ; \\
1467.56\end{array}$ & 0.92 & & IA \\
\hline \multirow[t]{2}{*}{$\mathrm{As}+\mathrm{Cd}$} & $\begin{array}{l}\text { GUTS-RED-SD-IA; } \\
\text { GUTS-RED-SD-DA }\end{array}$ & $\begin{array}{c}309.96 \\
334.98\end{array}$ & $\begin{array}{r}\mathbf{0 . 9 5} \\
0.8\end{array}$ & & IA \\
\hline & $\begin{array}{l}\text { GUTS-RED-IT-IA; } \\
\text { GUTS-RED-IT-DA }\end{array}$ & $\begin{array}{r}\text { 300.43; } \\
313.87\end{array}$ & $\begin{array}{r}\mathbf{0 . 9 7} \\
0.9\end{array}$ & & IA \\
\hline \multirow[t]{2}{*}{$\mathrm{IMI}+\mathrm{THI}$} & $\begin{array}{l}\text { GUTS-RED-SD-IA; } \\
\text { GUTS-RED-SD-DA }\end{array}$ & $\begin{array}{c}379.89 ; \\
251.78\end{array}$ & $\begin{array}{r}0.48 ; \\
\quad 0.8\end{array}$ & & DA \\
\hline & $\begin{array}{l}\text { GUTS-RED-IT-IA; } \\
\text { GUTS-RED-IT-DA }\end{array}$ & $\begin{array}{l}418.58 ; \\
\mathbf{2 5 2 . 2 9}\end{array}$ & $\begin{array}{r}0.46 ; \\
\mathbf{0 . 8}\end{array}$ & & DA \\
\hline
\end{tabular}

${ }^{a}$ The simultaneous fits have been assessed with the likelihood-ratio test (significant at $p<0.05$, in bold font). The prediction efficiency is quantified with the AIC and $r^{2}$ value (smaller value for AIC and higher value for $r^{2}$, best in bold font).

the single-chemical data will show cases where the DA model can be excluded a priori, if the data are accurate enough, and repeatable. For situations where the DA model is a possibility, and without data on the mixture, both models should be considered from a risk assessment perspective.

For the effect of the mixture of MGK 264 and glyphosate on the survival of E. crypticus, two lines of evidence confirm that damage addition is not the appropriate model. The DA model fits for the two chemicals in single exposures were considerably worse than the fits with the IA model (Table 1). This can be attributed to the expectation that these two chemicals lead to different forms of damage, meaning that they do not share the mortality-related parameters (Figure 3). As a result, the simultaneous fit, forcing the product $m_{\mathrm{w}} \times b_{\mathrm{w}}$ to be the same, led to a poor fit on the data. Finally, the DA model 
A) Calibration of the single exposures with the GUTS-RED-SD damage addition model
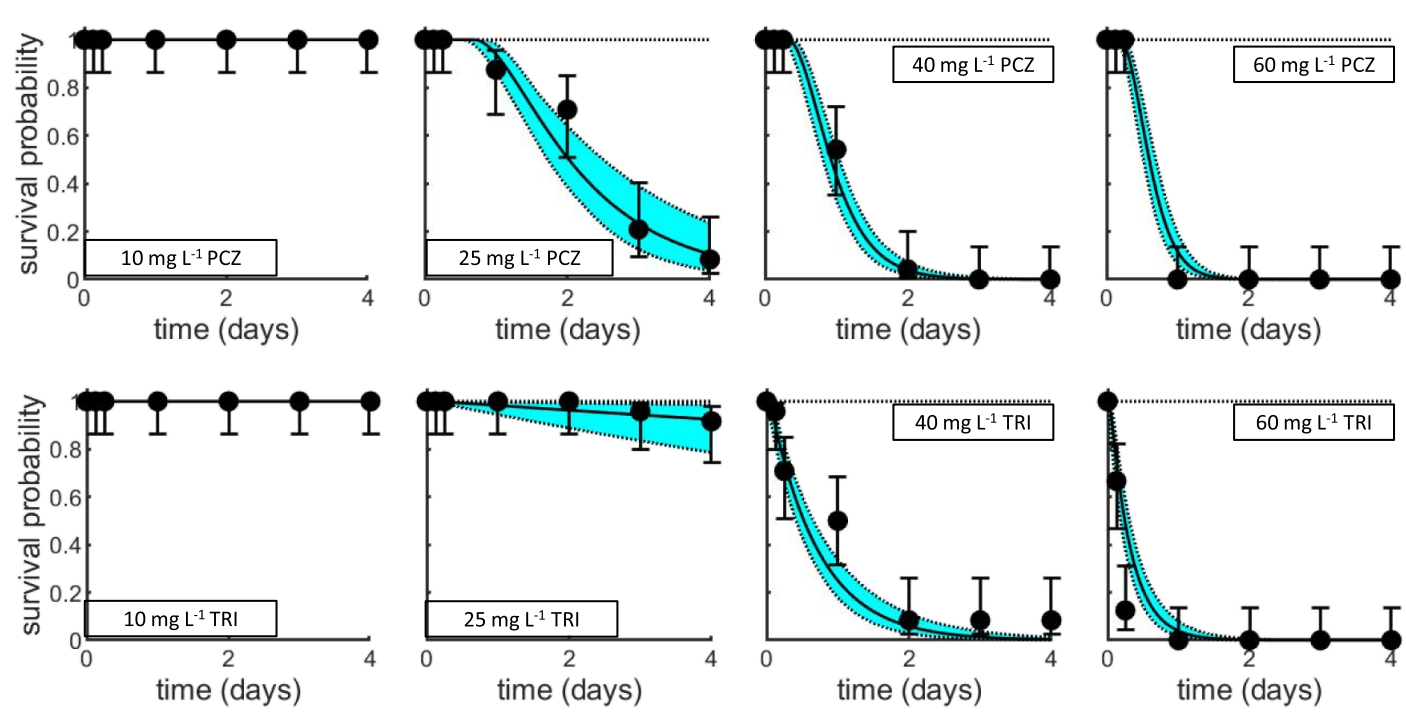

B) Prediction of the mixture exposure with the GUTS-RED-SD damage addition model
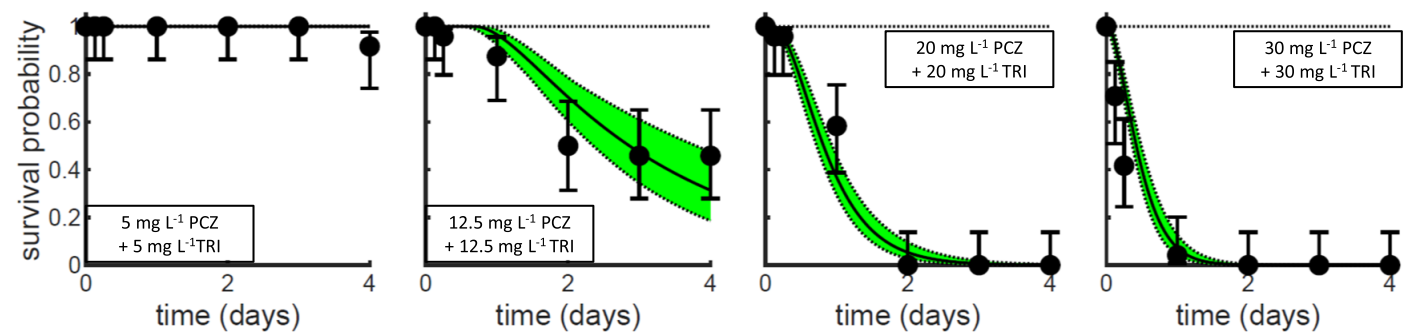

Figure 4. Observed and simulated survival over time of E. crypticus exposed to prochloraz (PCZ) and triflumizole (TRI) as a single exposure (A) and in mixture (B). The two top rows of plots (panel A) show the calibration of the GUTS-RED-SD damage addition model to the survival in the single exposures. The bottom row of plots (panel B) shows the prediction of the mixture effect using the model parameters that resulted from calibration with the single exposures. Observed fractions of survivors (points, bars show Wilson score confidence intervals) are overlaid with model simulations (solid lines, confidence intervals as a blue area for the fit and a green area for the prediction). The dashed lines are the background mortality, fitted to the control treatment.

provided a poor prediction of the mixture effects (Figure S4 and Table 1), compared to the IA model (Figure 5B, Figure S3B, and Table 1).

The first step of reanalyzing data from the literature with the GUTS-RED mixture model followed the same workflow to choose between DA and IA by looking first at the mortalityrelated parameters (Figure 3). The plotting of the mortality parameters for arsenic and cadmium for the A. mellifera dataset suggested use of the IA model as the most appropriate choice, and it indeed provided significantly better fits of the survival effect in the single exposures than did the DA model (Table 1). This result was in accordance with the hypothesis of dissimilar mode of action of these two metals. ${ }^{32}$ Further, the prediction of the mixture effect was slightly better with the IA model (Table 1, Figure 6, and Figure S5). Based on these data, Robinson et al. ${ }^{32}$ concluded that the classic IA model provided a slightly better prediction of the mixture than the CA model, which is in accordance with our TKTD modeling results.

Imidacloprid and thiacloprid are both systemic neonicotinoids acting on the acetylcholine receptor (nAChR) in $D$. magna. Therefore, the DA model was likely appropriate to predict the joint effect of this mixture on D. magna. This hypothesis was confirmed with (i) the plot of product of $m_{\mathrm{w}}$, $b_{\mathrm{w}}$, and $F_{\mathrm{s}}$ parameters (Figure 3), (ii) the simultaneous fits of the single exposures (Table 1), and (iii) the prediction of the mixture, which was better with the DA model (Table 1 and Figures S6 and S7). Loureiro et al. ${ }^{34}$ used the classical mixture approach to test the mixture effect of these two neonicotinoids and identified a potential synergistic interaction. Our TKTD approach supports the analysis as there is an underestimation of the predicted effect with both DA and IA models. This observation is consistent with additional toxicity beyond that which either model, damage addition (DA) or survival probability multiplication (IA), can account for (Figures S6 and S7).

Low Dose Effects Is the Key to Assess Mixture Effect. An important finding is that the differences in predictions between the DA and IA models appear mainly when chemicals are applied at low concentrations in the mixture, even below the threshold for the effects of single exposures. Considering low concentrations of prochloraz and triflumizole in mixture (e.g., $12.5 \mathrm{mg} \mathrm{L}^{-1}$ ), the DA model provided a very accurate prediction of the mixture (Figure 4), while in contrast, the IA model predicted no effect (Figure S2). This clear difference contrasts with that at higher concentrations, where even though the prediction of the DA model was better, the IA model was still able to provide an adequate prediction of the effect close to that for the DA model (Figure S2). We observed the same pattern of difference at low concentrations and near similarity at high concentrations for the DA and IA predictions 
A) Calibration of the single exposures with the GUTS-RED-SD independent action model
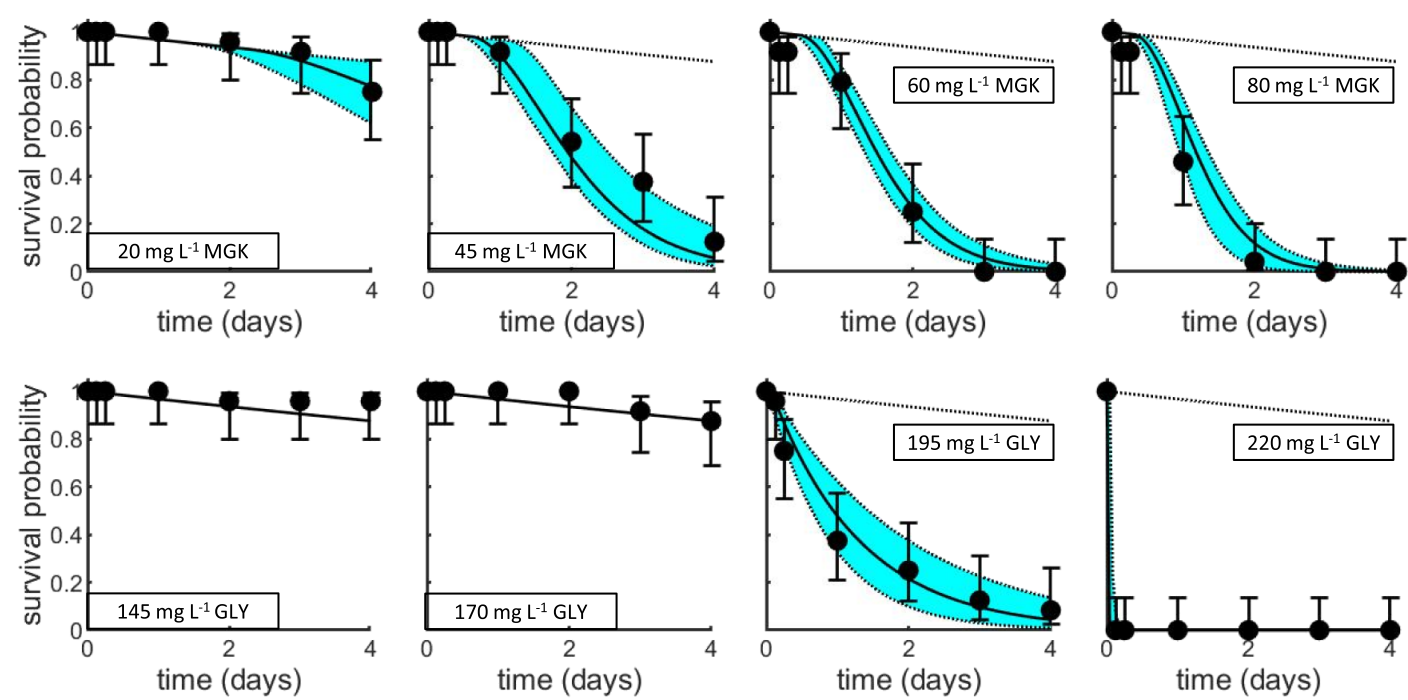

B) Prediction of the mixture exposure with the GUTS-RED-SD independent action model
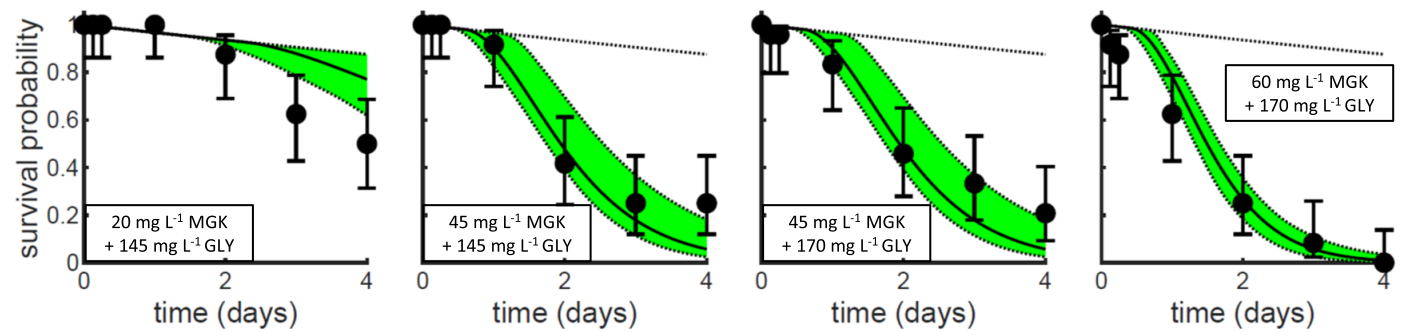

Figure 5. Observed and simulated survival over time of E. crypticus exposed to MGK 264 and glyphosate as a single exposure (A) and in mixture (B). The two top rows of plots (A) show the calibration of the GUTS-RED-SD independent action model to the survival in the single exposures. The bottom row of plots (panel B) shows the prediction of the mixture effect using the model parameters that resulted from calibration with the single exposures. Observed fractions of survivors (points, bars show Wilson score confidence intervals) are overlaid with model simulations (solid lines, confidence intervals as a blue area for the fit and a green area for the prediction). The dashed lines are the background mortality, fitted to the control treatment.
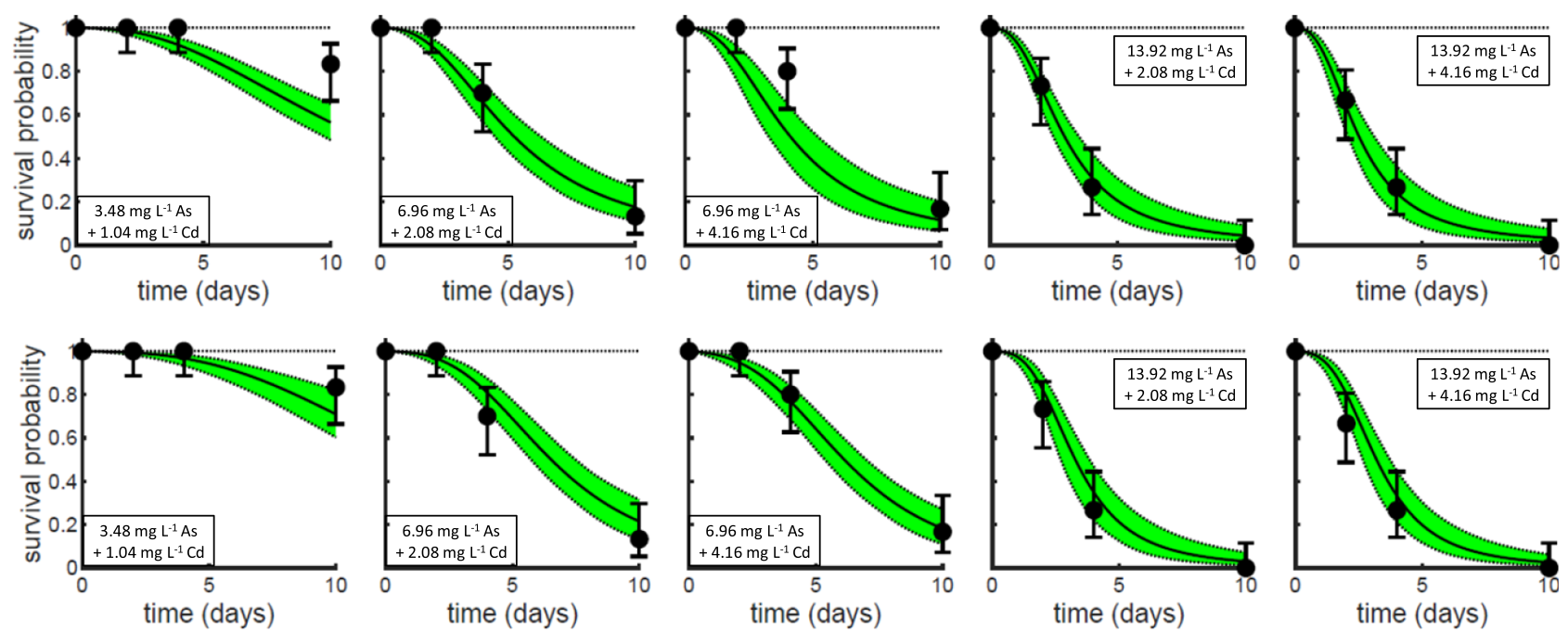

Figure 6. Observed and predicted survival over time of A. mellifera exposed to arsenic (As) and cadmium (Cd) in mixture with the GUTS-RED-IT damage addition model (top row of plots) or with the independent action model (bottom row of plots). Observed fractions of survivors (points, bars show Wilson score confidence intervals) are overlaid with model simulations (solid lines, confidence intervals as a green area). The dashed lines are the background mortality, fitted to the control treatment. Data from Robinson et al. ${ }^{32}$

for the effect of the arsenic and cadmium mixture on $A$. mellifera. Thus, the differences between the prediction of the
DA and IA models on this dataset were predominantly found at low concentration $\left(3.48 \mathrm{mg} \mathrm{L}^{-1}\right.$ As and $1.04 \mathrm{mg} \mathrm{L}^{-1} \mathrm{Cd}$; 
Figure 6 and Figure S5). The same observation was made with the prediction of the effect of imidacloprid and thiacloprid in mixture on D. magna, for which DA provided a better prediction at low concentration, but the prediction of both models at high concentrations was equivalent (Figures S6 and S7). One consequence of these findings is that when analyzing the mixture effect, the low-medium concentration effects contain more information, while the information about the mode of action can be obscured in the data from the higher tested concentrations. This finding should be considered not only to understand the impact of chemicals on a nontarget organism but also because low exposure concentration is the regular scenario in the field. ${ }^{37}$

Advantages, Usefulness, and Limits of the GUTS-RED Mixture Models. The two dynamic GUTS-RED mixture models presented in this study bring new tools to investigate whether chemicals lead to the same or different forms of damage and therefore which model is more appropriate (DA or IA) for predicting mixture effects. First, comparing the GUTS model parameters of each toxicant to see if there are overlaps (Figure 3) provides a new diagnostic tool to quickly assess if the toxicants are likely to produce the same form of damage. This information is used within the model framework to select the most appropriate mixture model out of DA or IA but also has a wider value for application in mechanistic toxicology. Within this framework, both the SD and IT approaches are complementary and need to be considered. If both show no overlaps, then it is unlikely that the chemicals lead to the same form of damage and the IA model is recommended. In contrast, if overlaps are found with the SD or IT approach only (e.g., prochloraz and triflumizole; Figure 3) or both (e.g., imidacloprid and thiacloprid; Figure 3), then the DA model should be considered as well. Second, the assessment of the simultaneous fits with the DA model compared to the IA model with the predictions can be used to support conclusions on the mode of action of the chemicals in the mixture relating to whether they cause effects through the same or dissimilar forms of damage.

The predictions of the model were especially good for our dedicated experiments with E. crypticus (Figures 4 and 5) but were also possible when using literature data from experiments with $D$. magna and A. mellifera, which were not specifically designed for this modeling approach (Figure 6 and Figures S5-S7). We here focused on binary mixtures and the two different models showed that according to the mode of action of the chemicals involved, they lead to very different predictions (Figure $4 \mathrm{~B}$ and Figure $\mathrm{S} 1 \mathrm{~B}$ vs Figure S2, and Figure 5B and Figure S3B vs Figure S4), highlighting that two models are required to predict binary mixture effects over time. The approach can be, in theory, extended to multiple compounds and it would work in the exact same manner: the similar compounds are added with weight factors (one weight factor less than the number of similar compounds) to yield a prediction of survival probability over time. The effects of groups of similar compounds are then combined with effects of any remaining dissimilar compounds into an IA analysis. An extensive program of work would be needed to underpin any such developments, with dedicated experiments, to explore the predictive power of the GUTS mixture model on multiple mixtures involving chemicals with the same and dissimilar mode of action.

The classic approach for predictive mixture hazard assessment is largely based on the concentration addition and independent action models using information derived from single time-point dose-response curves, thus ignoring the time dimension. The time dimension can only be included into the classic descriptive approach by repeatedly fitting the doseresponse model at every time point, which would require many more parameters in total to analyze experimental datasets. Such an analysis does not help us understand the processes underlying the mixture response and therefore does not allow us to predict effects due to exposure scenarios that are untested in the lab (e.g., longer timescales or changing mixture ratios through time). GUTS provides this possibility because GUTS models have biologically meaningful parameters that can be used to analyze and predict mixture effects over time. This is important because we know that chemicals can differ substantially in their toxicokinetics and/or toxicodynamics and this can have implications for the resulting time course of the mixture effect. In contrast to other mixture effect models, the GUTS model can deal with mixtures that vary in concentration over time or when chemicals are applied sequentially, which can lead to very different effects (e.g., Figure S8). ${ }^{15}$ Hence, our proposed framework provides a process-based simulation that is more mechanistically based and more widely applicable than conventional dose-response approaches. Once calibrated, a GUTS model with a few parameters can be applied on real, time-variable mixture exposure scenarios without the need for additional experimental work. ${ }^{38}$

TKTD models are powerful tools for the prediction of mixture toxicity as it has been previously demonstrated. ${ }^{39-41}$ However, the previous TKTD models were species-dependent, such as PBPK models designed for specific chemicals, while our approach is generic and will work with very much standard toxicological data. Furthermore, because GUTS unified all previous TKTD models for survival, our GUTS mixture model also presents a unification of the mixture models based on those previous TKTD models for survival. ${ }^{10,14,17}$ Risk assessment needs such a generic approach, applicable in an efficient way to different organisms and thousands of chemicals in the environment. ${ }^{1}$ The European Food Safety Authority (EFSA) recently recognized the GUTS framework as ready to use in ERA, ${ }^{23}$ and we here formalized for the first time the GUTS framework for mixture toxicity assessment.

Potentially moving beyond straightforward additive or independent effects, the GUTS-RED mixture models can also be a suitable tool to find interactions in mixtures. These occur when the model prediction deviates from the mixture effect observed over time, as with the data from Loureiro et al. ${ }^{34}$ When such interactions are found, the classical approach cannot provide mechanistic explanations of the synergism or antagonism, while a GUTS model has the potential to achieve this understanding (although that goes beyond the models presented in this study). For example, Cedergreen et al. ${ }^{42}$ highlighted that the biotransformation of the insecticide cypermethrin was reduced in the presence of the fungicide propiconazole, leading to a higher concentration of cypermethrin in D. magna, leading to stronger effects in combined exposure. The modeling of these data with a full GUTS model provided a good prediction of the mixture effect, accounting for the interaction between the two chemicals. GUTS-RED models can be refined to predict the joint effect of mixtures with interaction. The interaction can involve toxicokinetic and/or toxicodynamic processes, ${ }^{43}$ and when the mechanisms of these interactions are understood, they can be incorporated 
into TKTD models. Such next-generation TKTD mixture effect models will also be able to accurately predict mixture effects with interactions. In this way, synergism and antagonism will be recognized as artifacts of underdeveloped null models ${ }^{44}$ and can be seen as an incentive for model improvement based on mechanistic underpinning, instead of the final result of an analysis.

\section{ASSOCIATED CONTENT}

\section{(s) Supporting Information}

The Supporting Information is available free of charge at https://pubs.acs.org/doi/10.1021/acs.est.0c05282.

E. crypticus culture; model equations; concentration table; IT approach for the dedicated experiments; plots of mixture predictions for the data extracted from the literature; tables of the parameter values and the table of the models' efficiency; simulation of two different sequential exposures; raw data (PDF)

\section{AUTHOR INFORMATION}

\section{Corresponding Author}

Sylvain Bart - Department of Environment and Geography, University of York, York YO10 5NG, U.K.; UK Centre for Ecology and Hydrology, Wallingford OX10 8BB, Oxfordshire, U.K.; (1) orcid.org/0000-0002-0290-5546; Email: sylvain.bart@york.ac.uk

\section{Authors}

Tjalling Jager - DEBtox Research, Stevensweert 6107, The Netherlands; O orcid.org/0000-0002-4424-1442

Alex Robinson - UK Centre for Ecology and Hydrology, Wallingford OX10 8BB, Oxfordshire, U.K.

Elma Lahive - UK Centre for Ecology and Hydrology, Wallingford OX10 8BB, Oxfordshire, U.K.

David J. Spurgeon - UK Centre for Ecology and Hydrology, Wallingford OX10 8BB, Oxfordshire, U.K.; (1) orcid.org/ 0000-0003-3264-8760

Roman Ashauer - Department of Environment and Geography, University of York, York YO10 5NG, U.K.; Syngenta Crop Protection AG, Basel 4058, Switzerland; (1) orcid.org/0000-0002-9579-8793

Complete contact information is available at: https://pubs.acs.org/10.1021/acs.est.0c05282

\section{Notes}

The authors declare the following competing financial interest(s): Roman Ashauer is employed by Syngenta Crop Protection AG, which sells some of the substances studied here.

\section{ACKNOWLEDGMENTS}

We thank Susana Loureiro for providing the raw data of the $D$. magna experiments. This work was supported by the Natural Environment Research Council (grant numbers NE/S00135/1 and $\mathrm{NE} / \mathrm{S} 00224 / 2)$. We also thank the journal reviewers because their comments helped us to improve the manuscript substantially.

\section{REFERENCES}

(1) Escher, B. I.; Stapleton, H. M.; Schymanski, E. L. Tracking complex mixtures of chemicals in our changing environment. Science 2020, 367, 388 .
(2) van Leeuwen, C. J.; Vermeire, T. G., Eds. Risk assessment of chemicals - an introduction; 2nd ed.; Springer: Dordrecht, The Netherlands, 2007.

(3) Ballabio, C.; Panagos, P.; Lugato, E.; Huang, J.-H.; Orgiazzi, A.; Jones, A.; Fernández Ugalde, O.; Borrelli, P.; Montanarella, L. Copper distribution in European topsoils: An assessment based on LUCAS soil survey. Sci. Total Environ. 2018, 636, 282-298.

(4) Boxall, A. B. A. Veterinary medicines and the environment. In: Comparative and Veterinary Pharmacology; Cunningham, F., Elliott, J., Lees, P., Eds.; Springer Berlin Heidelberg: 2010; pp. 291-314

(5) Backhaus, T.; Faust, M. Predictive environmental risk assessment of chemical mixtures: a conceptual framework. Environ. Sci. Technol. 2012, 46, 2564-2573.

(6) Baas, J.; Jager, T.; Kooijman, B. Understanding toxicity as processes in time. Sci. Total Environ. 2010, 408, 3735-3739.

(7) Péry, A. R. R.; Bedaux, J. J. M.; Zonneveld, C.; Kooijman, S. A. L. $\mathrm{M}$. Analysis of bioassays with time-varying concentrations. Water Res. 2001, 35, 3825-3832.

(8) Ashauer, R.; Escher, B. I. Advantages of toxicokinetic and toxicodynamic modelling in aquatic ecotoxicology and risk assessment. J. Environ. Monit. 2010, 12, 2056-2061.

(9) Bart, S.; Pelosi, C.; Nélieu, S.; Lamy, I.; Péry, A. R. R. An energybased model to analyze growth data of earthworms exposed to two fungicides. Environ. Sci. Pollut. Res. 2020, 27, 741-750.

(10) Ashauer, R.; Boxall, A. B. A.; Brown, C. D. Modeling combined effects of pulsed exposure to carbaryl and chlorpyrifos on Gammarus pulex. Environ. Sci. Technol. 2007, 41, 5535-5541.

(11) Baas, J.; van Houte, B. P. P.; van Gestel, C. A. M.; Kooijman, S. A. L. M. Modeling the effects of binary mixtures on survival in time. Environ. Toxicol. Chem. 2007, 26, 1320-1327.

(12) Baas, J.; Jager, T.; Kooijman, B. A review of DEB theory in assessing toxic effects of mixtures. Sci. Total Environ. 2010, 408, $3740-3745$.

(13) Baas, J.; Stefanowicz, A. M.; Klimek, B.; Laskowski, R.; Kooijman, S. A. L. M. Model-based experimental design for assessing effects of mixtures of chemicals. Environ. Pollut. 2010c, 158, 115-120.

(14) Baas, J.; Jager, T.; Kooijman, S. A. L. M. A model to analyze effects of complex mixtures on survival. Ecotoxicol. Environ. Saf. 2009, $72,669-676$.

(15) Ashauer, R.; O'Connor, I.; Escher, B. I. Toxic mixtures in timethe sequence makes the poison. Environ. Sci. Technol. 2017, 51, 30843092.

(16) Lee, J. H.; Landrum, P. F.; Koh, C.-h. Prediction of timedependent PAH toxicity in Hyalella azteca using a damage assessment model. Environ. Sci. Technol. 2002, 36, 3131-3138.

(17) Lee, J.-H.; Landrum, P. F. Development of a multi-component damage assessment model (MDAM) for time-dependent mixture toxicity with toxicokinetic interactions. Environ. Sci. Technol. 2006, 40, 1341-1349.

(18) Jager, T.; Albert, C.; Preuss, T. G.; Ashauer, R. General Unified Threshold model of Survival - a toxicokinetic-toxicodynamic framework for ecotoxicology. Environ. Sci. Technol. 2011, 45, 2529-2540.

(19) Jager, T.; Ashauer, R. Modelling survival under chemical stress. A comprehensive guide to the GUTS framework; Toxicodynamics Ltd.: York, U.K., 2018.

(20) Zitko, V. An equation of lethality curves in tests with aquatic fauna. Chemosphere 1979, 8, 47-51.

(21) Ashauer, R.; O'Connor, I.; Hintermeister, A.; Escher, B. I. Death Dilemma and Organism Recovery in Ecotoxicology. Environ. Sci. Technol. 2015, 49, 10136-10146.

(22) Jager, T. Revisiting simplified DEBtox models for analysing ecotoxicity data. Ecol. Model. 2020, 416, 108904.

(23) EFSA Panel on Plant Protection Products and their Residues (PPR); Ockleford, C.; Adriaanse, P.; Berny, P.; Brock, T.; Duquesne, S.; Grilli, S.; Hernandez-Jerez, A. F.; Bennekou, S. H.; Klein, M.; Kuhl, T.; Laskowski, R.; Machera, K.; Pelkonen, O.; Pieper, S.; Smith, R. H.; Stemmer, M.; Sundh, I.; Tiktak, A.; Topping, C. J.; Wolterink, G.; Cedergreen, N.; Charles, S.; Focks, A.; Reed, M.; Arena, M.; Ippolito, A.; Byers, H.; Teodorovic, I. Scientific Opinion on the state of the art 
of Toxicokinetic/Toxicodynamic (TKTD) effect models for regulatory risk assessment of pesticides for aquatic organisms. EFSA J. 2018, 16, No. e05377.

(24) Villeneuve, D. L.; Blankenship, A. L.; Giesy, J. P. Derivation and application of relative potency estimates based on in vitro bioassay results. Environ. Toxicol. Chem. 2000, 19, 2835-2843.

(25) Van den Berg, M.; Birnbaum, L. S.; Denison, M.; De Vito, M.; Farland, W.; Feeley, M.; Fiedler, H.; Hakansson, H.; Hanberg, A.; Haws, L.; Rose, M.; Safe, S.; Schrenk, D.; Tohyama, C.; Tritscher, A.; Tuomisto, J.; Tysklind, M.; Walker, N.; Peterson, R. E. The 2005 World Health Organization reevaluation of human and mammalian toxic equivalency factors for dioxins and dioxin-like compounds. Toxicol. Sci. 2006, 223-241.

(26) Chèvre, N.; Loepfe, C.; Singer, H.; Stamm, C.; Fenner, K.; Escher, B. I. Including mixtures in the determination of water quality criteria for herbicides in surface water. Environ. Sci. Technol. 2006, 40, 426-435.

(27) Lee, Y.; Escher, B. I.; von Gunten, U. Efficient removal of estrogenic activity during oxidative treatment of waters containing steroid estrogens. Environ. Sci. Technol. 2008, 42, 6333-6339.

(28) Ankley, G. T.; Bennett, R. S.; Erickson, R. J.; Hoff, D. J.; Hornung, M. W.; Johnson, R. D.; Mount, D. R.; Nichols, J. W.; Russom, C. L.; Schmieder, P. K.; Serrrano, J. A.; Tietge, J. E.; Villeneuve, D. L. Adverse outcome pathways: a conceptual framework to support ecotoxicology research and risk assessment. Environ. Toxicol. Chem. 2010, 29, 730-741.

(29) Roembke, J.; Knacker, T. Aquatic toxicity test for enchytraeids. Hydrobiologia 1989, 180, 235-242.

(30) Fungicide resistance action committee (FRAC). FRAC Code List (C) 2020: Fungal control agents sorted by cross resistance pattern and mode of action; https://www.frac.info/knowledge-database/ knowledge-database.

(31) Castro-Ferreira, M. P.; de Boer, T. E.; Colbourne, J. K.; Vooijs, R.; van Gestel, C. A. M.; van Straalen, N. M.; Soares, A. M. V. M.; Amorim, M. J. B.; Roelofs, D. Transcriptome assembly and microarray construction for Enchytraeus crypticus, a model oligochaete to assess stress response mechanisms derived from soil conditions. BMC Genomics 2014, 15, 302.

(32) Robinson, A.; Hesketh, H.; Lahive, E.; Horton, A. A.; Svendsen, C.; Rortais, A.; Dorne, J. L.; Baas, J.; Heard, M. S.; Spurgeon, D. J. Comparing bee species responses to chemical mixtures: common response patterns? PLoS One 2017, 12, No. e0176289.

(33) Hesketh, H.; Lahive, E.; Horton, A. A.; Robinson, A. G.; Svendsen, C.; Rortais, A.; Dorne, J.-L.; Baas, J.; Spurgeon, D. J.; Heard, M. S. Extending standard testing period in honeybees to predict lifespan impacts of pesticides and heavy metals using dynamic energy budget modelling. Sci. Rep. 2016, 6, 37655.

(34) Loureiro, S.; Svendsen, C.; Ferreira, A. L. G.; Pinheiro, C.; Ribeiro, F.; Soares, A. M. V. M. Toxicity of three binary mixtures to Daphnia magna: Comparing chemical modes of action and deviations from conceptual models. Environ. Toxicol. Chem. 2010, 29, 17161726.

(35) Brown, L. D.; Tony, C. T.; Das Gupta, A. Interval Estimation for a Binomial Proportion. Stat. Sci. 2001, 16, 101-133.

(36) Jager, T. Robust likelihood-based approach for automated optimisation and uncertainty analysis of toxicokinetic-toxicodynamic models. Integr. Environ. Assess. Manag. 2020, DOI: 10.1002/ ieam.4333, in press.

(37) Silva, V.; Mol, H. G. J.; Zomer, P.; Tienstra, M.; Ritsema, C. J.; Geissen, V. Pesticide residues in European agricultural soils - a hidden reality unfolded. Sci. Total Environ. 2019, 653, 1532-1545.

(38) Arlos, M. J.; Focks, A.; Hollender, J.; Stamm, C. Improving Risk Assessment by Predicting the Survival of Field Gammarids Exposed to Dynamic Pesticide Mixtures. Environ. Sci. Technol. 2020, 54, 1238312392.

(39) Abbas, R.; Hayton, W. L. A physiologically based pharmacokinetic and pharmacodynamic model for paraoxon in rainbow trout. Toxicol. Appl. Pharmacol. 1997, 145, 192-201.
(40) Tebby, C.; Brochot, C.; Dorne, J.-L.; Beaudouin, R. Investigating the interaction between melamine and cyanuric acid using a Physiologically-Based Toxicokinetic model in rainbow trout. Toxicol. Appl. Pharmacol. 2019, 370, 184-195.

(41) Timchalk, C.; Poet, T. S. Development of a physiologically based pharmacokinetic and pharmacodynamic model to determine dosimetry and cholinesterase inhibition for a binary mixture of chlorpyrifos and diazinon in the rat. Neurotoxicology 2008, 29, 428443.

(42) Cedergreen, N.; Dalhoff, K.; Li, D.; Gottardi, M.; Kretschmann, A. C. Can toxicokinetic and toxicodynamic modeling be used to understand and predict synergistic interactions between chemicals? Environ. Sci. Technol. 2017, 51, 14379-14389.

(43) Spurgeon, D. J.; Jones, O. A. H.; Dorne, J.-L. C. M.; Svendsen, C.; Swain, S.; Stürzenbaum, S. R. Systems toxicology approaches for understanding the joint effects of environmental chemical mixtures. Sci. Total Environ. 2010, 408, 3725-3734.

(44) Schäfer, R. B.; Piggott, J. J. Advancing understanding and prediction in multiple stressor research through a mechanistic basis for null models. Global Change Biol. 2018, 24, 1817-1826. 